“C 2007 IEEE. Personal use of this material is permitted. Permission from IEEE must be obtained for all other uses, in any current or future media, including reprinting/republishing this material for advertising or promotional purposes, creating new collective works, for resale or redistribution to servers or lists, or reuse of any copyrighted component of this work in other works." 


\title{
Rank-Deficiency in Indoor MIMO
}

\author{
Vu Van Khang \\ College of Technology - Vietnam National University Hanoi, Vietnam \\ Nguyen Dinh Thong \\ Faculty of Engineering - University of Technology Sydney, Australia
}

\begin{abstract}
This paper points out in an analytical way that rankdeficiency in indoor MIMO is typically due to the small size of scattering windows in the NLOS propagation path between the transmitter and the receiver.
\end{abstract}

\section{INTRODUCTION}

Early research efforts in MIMO communications have concentrated on the linear gain of the system data rate obtained from scattering-rich multipath fading conditions which fully decorrelate antenna elements in the same array from one another. This is mainly because of the underlining mathematical attractiveness of the linear gain conditions. As a consequence, analytical results for optimal system capacity are available mostly for MIMO channels that exhibit independent and identically distributed (i.i.d.) purely Rayleigh and flat fading (i.e. frequency non-selective) [1], [2]. In this ideal condition the system capacity is proportional to the minimum of the numbers of antenna elements in transmit and receive arrays, which is the full rank of the system matrix. For correlated fading and non-Rayleigh cases, including Ricean fading, the optimal linear MIMO gain is usually not achievable, and studies have used a mainly simulation and experimental approach because of the mathematical complexity involved. High rank channels are achieved when antennas in the same array are significantly decorrelated from one another, hence rendering full spatial multiplexing gain through orthogonal eigenmode channels, i.e. independent parallel data 'pipes'. This usually occurs either in the presence of rich multipath scatterers which tend to destroy any spatial fading correlation, or when antennas in the array are adequately separated from one another.

In most existing publications on MIMO, high or full ranks are assumed to be the result of a dense or rich scattering environment. Although it has not been explicitly stated, the assumption refers to local scattering around end antennas. In most urban environments, this assumption is often valid except in some cases of roof-top diffraction [3]. But in crowded urban areas and indoor environments, rankdeficiency of the channel matrix can occur under perfect i.i.d

This work is supported by Research Project \# QG-TD0609 from the Vietnam National University Hanoi.
Rayleigh conditions where antennas are sufficiently decorrelated by nearby scatterers. Thus rich scattering is only a necessary but not sufficient condition for high rank MIMO channels, and rank-deficiency and spatial fading correlation are not necessarily related. MIMO channels with uncorrelated spatial fading at both the transmitter and receiver but having a degenerate rank one are referred to as "pinhole or keyhole" channels [5]. In most practical cases, rank-deficiency cannot be explained from the classical singlescattering model. Rank-deficiency is related to the structure of scattering in the propagation paths, e.g. diffraction over windows or table tops and tunnelling/waveguiding along corridors [4]. Recently a double-scattering MIMO model has been proposed in which the channel matrix is characterized by a product of two statistically independent complex Gaussian matrices - one for each end [5]. This doublescattering model can decouple (i.e. can capture separately) the effects of rank-deficiency and spatial fading correlation in MIMO channels. The model thus allows us to study a wide variety of propagation environments ranging from pure Rayleigh (or full rank) to pure keyhole/pinhole (or rank one). Between these two extreme cases there are other scenarios in which the system degenerates not right to rank one but to less than full rank, due to the lack of scattering richness. Strictly speaking, correlated rank one case and uncorrelated low rank case (but not rank one) are not keyhole/pinhole.

In practice, spatial correlation can arise from various reasons such as inadequate antenna element spacing or correlated scattering from nearby scatterers. Most studies on characteristics of spatial fading correlation use a large number of channel realisations either through field measurements, or through ray-tracing simulations of the actual physical propagation environment using the Monte Carlo technique. A more flexible and far less demanding, yet more informative approach is to construct a geometric scatterer model that can approximately describe the scattering wireless environment of interest and then use ray-tracing to calculate the path gain correlations [6], [7]. The "one-ring" model was first used in [6] and later by others [7] to study fading correlation in fixed wireless communication scenarios in which the base station is usually elevated and unobstructed while mobile stations are frequently surrounded by local scatterers. 
In indoor MIMO environments, there are many typical scenarios that scatterers are relatively simple to model at least experimentally, e.g. a window or door in a wall between the transmitter and the receiver, or a corridor in an office building [4]. The authors in [4] have used these scatterer models in a controlled environment to study experimentally the distribution of the eigenvalues for three different channel setups: a small rectangular hole, a large square hole, and a small rectangular hole with waveguide attached. In this paper, to complement the experimental work in [4], we prove in an analytical way that the small size of the hole in the shielded separating wall is responsible for the rank deficiency of the indoor MIMO system, and that "keyhole/pinhole" condition is not a discrete state but is the result of a gradual rank reduction.

\section{SYSTEM MODEL AND CAPACITY}

\section{A. Uncorrelated Fading MIMO Channels}

A narrow band MIMO system of $M$ antennas in the receive linear array and $N$ antennas in the transmit linear array is described by the following equation

$\boldsymbol{y}=\mathbf{H} \boldsymbol{x}+\boldsymbol{n}$

where $\boldsymbol{y}$ is a $M \mathrm{x} 1$ complex column vector and $\mathrm{x}$ is a $N \mathrm{x} 1$ complex column vector representing signals at the receive and transmit antennas respectively, $\boldsymbol{n}$ is a $M \mathrm{x} 1$ complex column vector representing the additive white Gaussian noise (AWGN) each element of which has a zero mean and a variance of $\sigma_{n}{ }^{2}$. The entry $H_{m, n}$ of $\mathbf{H}$ represents the complex channel-fading coefficient from the $n$th transmit antenna to the $m$ th receive antenna, i.e arriving at $m$ from $n$. The transmission over the narrow band channel is assumed to be frequency flat (i.e. non-selective). When the fading is purely Rayleigh, the channel gain $H_{m, n}$ in this paper, is modelled as a circularly symmetric complex Gaussian random variable having zero mean and unit variance, denoted as $C \mathcal{N}(0,1)$.

For the case of uncorrelated signals between transmit antennas, we have the well known capacity expression given by [1], [2]

$$
C=\log _{2}\left[\operatorname{det}\left(\mathbf{I}_{J}+\frac{P}{N \sigma_{n}^{2}} \mathbf{W}_{\mathbf{H}}\right)\right] \mathrm{bps} / \mathrm{Hz}
$$

where $P$ is the total transmit power, $\mathbf{I}_{J}$ is the $J \mathrm{x} J$ identity matrix with $J=\min (M, N)$ which is the rank of $\mathbf{H}$, and

$\mathbf{W}_{\mathbf{H}}= \begin{cases}\mathbf{H H}^{H} & \text { if } J=M \leq N \\ \mathbf{H}^{H} \mathbf{H} & \text { if } J=N \leq M\end{cases}$

By using the eigen value decomposition (EVD), we have

$$
\mathbf{H} \mathbf{H}^{\mathrm{H}}=\mathbf{U} \boldsymbol{\Lambda} \mathbf{U}^{\mathrm{H}} \text { and } \mathbf{H}^{\mathrm{H}} \mathbf{H}=\mathbf{V} \boldsymbol{\Lambda} \mathbf{V}^{\mathrm{H}}
$$

where $\mathbf{U}$ is a $M \mathrm{x} M$ unitary matrix whose columns are the eigenvectors of $\mathbf{H H}^{\mathrm{H}}, \mathbf{V}$ is a $N \mathrm{x} N$ unitary matrix whose columns are the eigenvectors of $\mathbf{H}^{\mathrm{H}} \mathbf{H}$, and $\boldsymbol{\Lambda}=\operatorname{diag}\left(\lambda_{1}, \lambda_{2}\right.$, . $\left.\ldots, \lambda_{J}\right)$.

Note that $\mathbf{H}$ is normalised as $E\left[\|\mathrm{H}\|^{2}\right]=\sum_{j} \lambda_{j}=M N$ over all realisations, and that (2) attains the Shannon limit below only when $\mathbf{H}$ is full rank,

$\mathrm{C}=\sum_{j=1}^{J} \log _{2}\left(1+\frac{P}{N \sigma_{n}^{2}} \lambda_{j}\right)$

\section{B. Double Scattering MIMO Channel Model}

Let the system matrix $\mathbf{H}=\left[\boldsymbol{h}_{1}\left|\boldsymbol{h}_{2}\right| \ldots . . \mid \boldsymbol{h}_{N}\right]$, i.e. written in terms of its column vectors. Define the $M N \times 1$ vector $\operatorname{vec}(\mathbf{H})=\left(\boldsymbol{h}_{1}^{T}\left|\boldsymbol{h}_{2}^{T}\right| \ldots \ldots \mid \boldsymbol{h}_{N}^{T}\right)^{T}$ i.e. formed by stacking the column vectors of $\boldsymbol{H}$ under each other.

The covariance matrix of $\mathbf{H}$ which describes the full $M N \mathrm{x} M N$ mutual correlation values between all elements of the channel matrix, is defined as

$\boldsymbol{\Psi}_{H} \equiv \operatorname{cov}(\mathbf{H}) \equiv \operatorname{cov}(\operatorname{vec}(\mathbf{H}))=E\left[\operatorname{vec}(\mathbf{H}) \operatorname{vec}(\mathbf{H})^{H}\right]$

i.e. an $M N \mathrm{x} M N$ matrix. Thus $\operatorname{cov}(\mathbf{H})$ completely specifies the second-order statistics of the channel matrix $\mathbf{H}$.

In [4] a double scattering MIMO channel model was proposed which can capture both spatial fading correlation and rank deficiency effect. The channel matrix of the model can be written in a general form as

$$
H=\Psi_{R}^{1 / 2} G_{R} \Psi_{S}^{1 / 2} G_{T} \Psi_{T}^{1 / 2}
$$

in which $\boldsymbol{\Psi}_{\mathbf{R}}, \boldsymbol{\Psi}_{\mathbf{S}}$, and $\boldsymbol{\Psi}_{\mathbf{T}}$ are receive, scatterer, and transmit square matrices, respectively, and $\mathbf{G}_{\mathbf{R}}$ and $\mathbf{G}_{\mathbf{T}}$ are i.i.d Rayleigh fading matrices on receive and transmit sides, respectively. For uncorrelated fading at both transmit and receive ends, (7) reduces to the general "pinhole" condition, i.e. uncorrelated low rank, as

$$
H=G_{R} \Psi_{S}^{1 / 2} G_{T}
$$

When $\boldsymbol{\Psi}_{H} \equiv \operatorname{cov}(\mathbf{H})$ is calculated from a deterministic ray-tracing model of the scatterer, i.e. assuming that there is no spatial correlation at both arrays, it can be easily prove that the random instances of $\mathbf{H}$ in (8) is equivalent to

$$
\operatorname{vec}(\mathbf{H})=\mathbf{\Psi}_{H}^{1 / 2} \operatorname{vec}(\mathbf{G})
$$

where $\mathbf{G}$ is a $M \times N$ purely Rayleigh channel matrix, i.e containing i.i.d. $\mathcal{C} \mathcal{N}(0,1)$ entries, and $\boldsymbol{\Psi}_{H}^{1 / 2}$ can be obtained from $\quad \boldsymbol{\Psi}_{\mathbf{H}}=\boldsymbol{\Psi}_{H}^{1 / 2}\left(\boldsymbol{\Psi}_{H}^{1 / 2}\right)^{H}$ using the standard Cholesky factorization. 


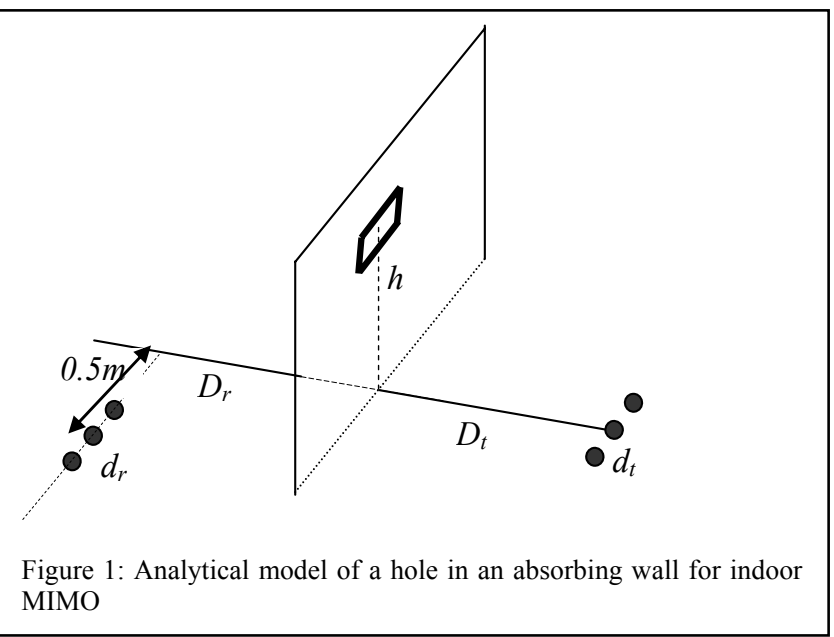

\section{ANALYTICAL MODEL OF A HOLE IN THE WALL}

As mentioned in the introduction, in this paper we propose a simple analytical model to complement the experimental study in [4] to study the rank deficiency phenomenon in an indoor MIMO environment. Figure 1 presents a typical geometry of a rectangular hole in a wall, very close to the setup in [4], i.e. the hole is the only possible propagation path between the transmitter and the receiver. The hole acts as a continuous rectangular ring of scatterers and LOS transmission is prevented by having the hole well elevated from the LOS. Rays from the transmitter are scattered by the edges of the hole to arrive at the receiver. With similar reasoning and assumptions as in [7], we modelled the dielectric phase shift by the scattering from the hole's edges as being uniformly distributed in $[-\pi, \pi]$ and i.i.d. with respect to the incidence angle. We therefore can ignore this scattering phase shift in the calculation of the covariance between the path gains.

By integrating around the hole's edges in the clockwise direction for the difference in the path phase shift between the two path $(i, p)$ and $(j, q)$, we arrive at the integral below for the path gain covariance:

$$
\begin{aligned}
& E\left[H_{i, p} H_{j, q}^{*}\right]
\end{aligned}
$$

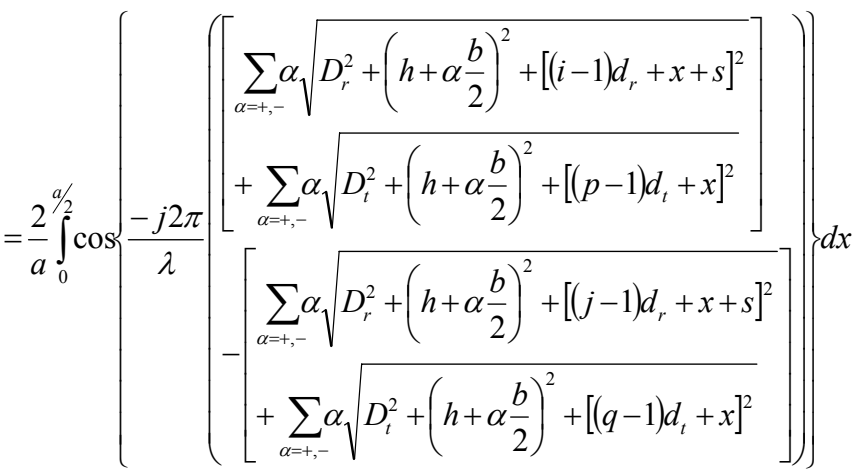

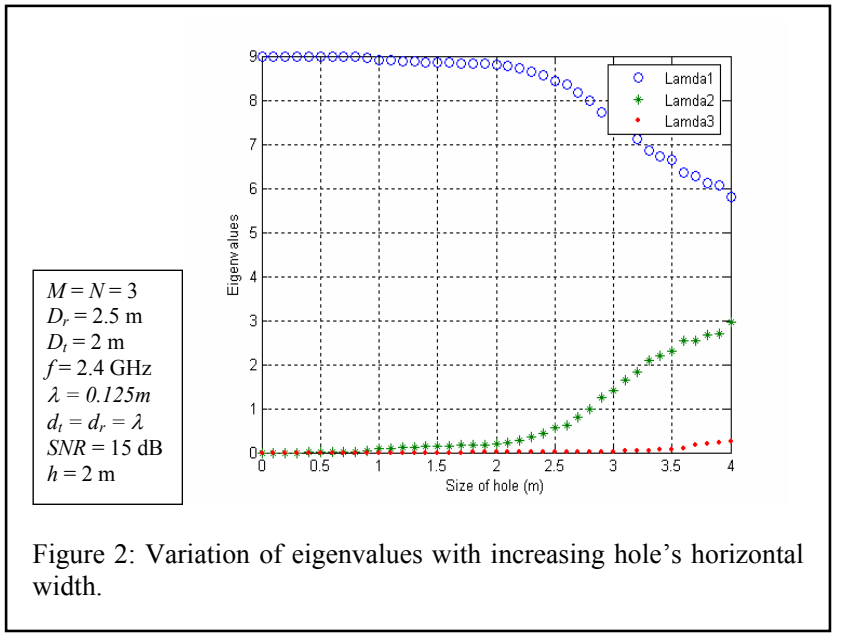

From (10) we obtain the $M N x M N$ covariance matrix in (6). We can then generate a large number of channel instances in (9) and hence the cumulative distribution of the eigenvalues of $\mathbf{H H}^{\mathrm{H}}$ and corresponding distribution for channel capacity. That is the approach used in [7] to study the spatial fading correlation due to local scatterers surrounding a subscriber unit. The local scatterers are modelled using the "one-ring" abstract model. However, since our objective in this paper is to study analytically the nature and the trend of rank deficiency of the indoor MIMO system due to a typical scattering structure in the propagation environment, we calculate the eigenvalues to prove that only the dominant eigenvalue survives as the hole size becomes too small.

\section{RESULT AND CONCLUSIONS}

The curves in Figures 2 and 3 represent the eigenvalues and corresponding system capacity, respectively, averaged over only 50 random instances of the channel for each hole size. The setup modelled in Figure 1 is very similar to the experimental setup in [4]. The lack of smoothness of the curves in Figures 2 and 3 is obviously due to the small number of random channel instances, due to a limitation in our computational resources. From the simulation results, shown in these figures, of our analytical scattering model of a hole in a wall of a typical indoor environment, it is clear that the channel rank gradually decreases to one (i.e. pure pinhole) as the size of the scattering hole decreases. We conclude that even under a perfectly uncorrelated fading condition between antenna elements at both ends, rank deficiency in a MIMO channel is controlled mainly by the structure of the scatterers in the propagation path. 


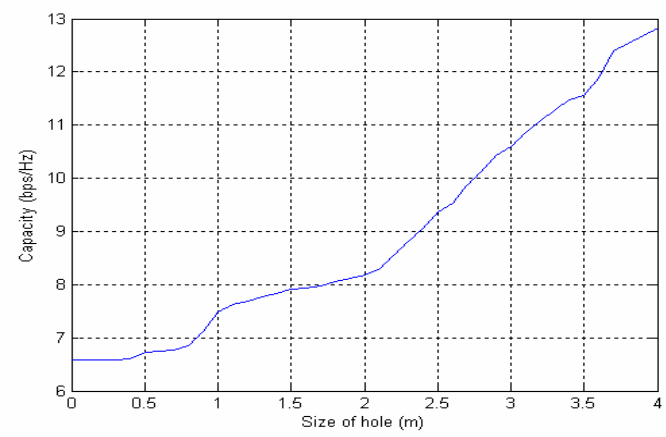

Figure 3: Variation of MIMO capacity with increasing hole's horizontal width

\section{REFERENCES}

[1] Emre Telatar, "Capacity of Multi-antenna Gaussian Channels," Eur. Trans. Telecommun., vol. 10, no. 6, Nov./Dec. 1999, pp. 585-596.

[2] G. J. Foschini and M.J. Gans, "On limits of wireless communications in a fading environment when using multiple antennas," Wireless Pers. Commun., vol. 6, Mar. 1998, pp.311-335.

[3] Dmitry Chizikh, Gerard J. Foschini, Michael J. Gans, and Reinaldo A. Valenzuela, "Keyholes, correlations, and capacities of multielement transmit and receive antennas," IEEE Trans. Wireless Commun., vol.1, pp. 361-368, Apr. 2002.

[4] Peter Almers, Fredrik Tufvesson, and Andreas F. Molisch, "Measurement of Keyhole Effect in a Wireless Multiple-Input Multiple-Output (MIMO) Channel," IEEE Communication Letters, vol.7, no. 8, pp.373-375, August 2003.

[5] David Gesbert, Helmut Bolcskei, Dhananjay A. Gore, and Arogyaswami J. Paulraj, "Outdoor MIMO Wireless Channels: Models and Performance Prediction," IEEE Transactions on Communications, vol. 50, no. 12, pp.1926-1934, Dec. 2002

[6] W. C.Jakes, Microwave Mobile Communications, Wiley, New York, 1974, pp. 60-65.

[7] Da-Shan Shiu, G. J. Foschini, M. J. Gans, and J. M. Kahn, "Fading Correlation and its Effect on the Capacity of Multielement Antenna Systems," IEEE Trans. Commun., vol.48, no. 3, pp. 502-513, Mar. 2000 